\title{
Système d'évaluation des terres à multiples échelles pour la détermination de l'impact de la gestion agricole sur la sécurité alimentaire au Katanga, R.D. Congo
}

\section{Emery Kasongo Lenge Mukonzo}

Promoteur: Eric Van Ranst (1)

Co-promoteurs: Michel Ngongo Luhembwe (2), Ann Verdoodt (1)

(I) Laboratoire des Sciences du sol, Université de Gand

(2) Faculté des Sciences Agronomiques, Université de Lubumbashi

Défense publique: 18 décembre 2008

L'insécurité alimentaire continue à sévir au Katanga malgré un immense potentiel pour le développement agricole. La plupart des denrées alimentaires de base sont importées des pays d'Afrique Australe, notamment la Zambie, le Zimbabwe et l'Afrique du Sud. Par contre, les travaux du Cadastre Minier viennent de porter à environ $72 \%$ de la superficie totale du Katanga l'étendue des terres attribuées sous forme de permis de recherche ou d'exploitation aux entreprises minières. Ce lotissement ne semble pas se préoccuper de la protection des bonnes terres constituant les meilleures opportunités de développement agricole de la province. Pourtant, ce développement est indispensable pour garantir la sécurité alimentaire de la population.

Cette nécessité a été à l'origine de la présente étude qui porte sur la mise au point d'un système d'évaluation des terres à plusieurs échelles permettant de déterminer l'impact de la gestion agricole sur la sécurité alimentaire au Katanga.

La première partie de cette étude porte sur l'analyse des ressources biophysiques de cette province et la simulation de l'évolution de la capacité de charge démographique des terres qui n'ont pas été cédées aux entreprises minières. Cette simulation a été faite sur une période de 50 ans (2008-2058) sous différents scénarios. Les résultats ont montré que d'ici 50 ans, les besoins alimentaires vont passer de 8, I x IO $^{12} \mathrm{Kcalories} \mathrm{à}_{56,8} \times \mathrm{IO}^{12}$ Kcalories ou plus par an alors que la capacité maximale de production des terres restantes a été estimée entre $34,44 \times 10^{12}$ et $68,87 \times 10^{12} \mathrm{Kcalories} \mathrm{par} \mathrm{an} \mathrm{avec} \mathrm{les} \mathrm{possibilités} \mathrm{éviden-}$ tes de décroissance graduelle due à la dégradation des terres. Ces terres sont incapables de nourrir à long terme la population du Katanga même si elles étaient toutes cultivées. Sur base de ces résultats, l'expansion minière a été confrontée avec les meilleures opportunités de développement agricole de la province. Les aires dominées par les groupes des sols jeunes et ceux qui sont relativement moins altérés, avec des bonnes conditions topographiques et une bonne disponibilité de l'eau ont été sélectionnées comme meilleures opportunités de développement agricole au Katanga. Il s'agit des plaines de la Lufira, la dépression de Kamalondo, le bassin de la Luapula-Moero et la région de KabongoManono-Nyunzu-Kongolo. De ces différentes régions sélectionnées, la proximité des 
plaines de la Lufira aux plus grandes agglomérations de la province, leur superficie totale qui avoisine I $000000 \mathrm{~d}$ 'ha et la nature de leurs terres ont montré qu'elles représentent un intérêt immédiat de valorisation agricole pour le renforcement de la production alimentaire locale. C'est au regard de cette nécessité que la présente étude a pris le soin d'y accorder le maximum d'attention.

L'analyse de la genèse des plaines de la Lufira a montré que la région des plaines supérieures est née de la traversée des eaux de la Lufira à travers une barrière naturelle, l'arc Lufilien, laissant à nu d'importants dépôts alluvionnaires par vidange progressif du lac de barrage qui s'était érigé dans cette région. Quant aux plaines moyennes, elles résultent de l'effondrement de la Grand Pénéplaine Miocène. De la période qui suit la formation de ce graben datent les importants dépôts lacustres et alluviaux au sein de la vallée. L'inventaire pédologique a montré la dominance du groupe des Fluvisols à côté des plages de Gleysols et Histosols dans les plaines alluviales de la Lufira supérieure, avec apparition du groupe des Luvisols au niveau de piedmont et celui des Acrisols sur les plateaux. Dans la moyenne Lufira, la couverture pédologique du paysage de la vallée alluviale est dominée par le groupe des Vertisols à côté des Gleysols et Fluvisols. Des Cambisols apparaissent dans le paysage de piedmont alors que les bandes de l'ancienne pénéplaine contiennent quelques plages de Ferralsols et d'Acrisols. Dans les deux plaines, des petites plages de Régosols sont observées sur les flancs rocheux entourant le paysage de la plaine.

Une analyse ethnopédologique à trois dimensions au sein de la communauté Bemba, ethnie dominante de la zone d'étude, a ressorti à la fois l'existence d'une systématique locale des sols quasi structurée, une connaissance empirique très poussée du comportement agricole des différents types des sols, et une perception cosmique des terres reliant leur productivité aux forces surnaturelles, notamment les esprits des ancêtres.

Un système d'évaluation des terres à multiples échelles a été ensuite élaboré pour permettre une évaluation des terres en quatre séquences permettant respectivement l'analyse de leur aptitude pour une agriculture pluviale, le degré d'aptitude des terres agricoles pour la culture du maïs, les rendements potentiels de ces terres à différents niveaux de gestion et la sensibilité des rendements du maïs aux différents niveaux des aménagements agricoles.

La première séquence a consisté dans l'élaboration d'un système paramétrique qui, sur base des scores attribués aux caractéristiques et qualités des terres a conduit à la séparation des terres aptes à l'exploitation agricole et celles qui ne le sont pas. Les problèmes de fertilité chimique et d'aération de la zone d'enracinement ont été identifiés comme principales contraintes à l'agriculture respectivement sur les Acrisols et les Fluvisols. La séquence suivante a consisté dans l'intégration des exigences de la culture du maïs au sein des arbres de décision par lesquels l'aptitude actuelle et potentielle des terres agricoles pour cette culture était dégagée. Les productions potentielles des terres pour la culture du maïs ont ensuite été calculées à deux niveaux de gestion après adaptation du modèle à trois niveaux hiérarchiques de prédiction des rendements au contexte socioéconomique des plaines supérieures de la Lufira. Une fonction logistique a été utilisée pour simuler l'indice de gestion de la culture tant en milieu paysan sur un Acrisol qu'en 
culture moderne sur un Fluvisol. Enfin, la sensibilité des rendements du maïs aux aménagements agricoles a été évaluée à travers une analyse des élasticités à l'issue de la mise au point des fonctions de production du type Cobb-Douglas. En culture traditionnelle du maïs comme en agriculture moderne, il a été observé que les aménagements agricoles engendrent des accroissements des rendements moins que proportionnels. L'analyse de la tendance du rendement par rapport aux variations de chaque type d'aménagement a été suggérée comme une approche permettant la sélection des niveaux les plus rentables d'aménagement de la culture. 